\title{
Exploration and Practice of Computational Thinking Training at University Computer Courses
}

\author{
Xie HongXia, He ban \\ School of Computer and Computing Science, Zhejiang University City College Hangzhou, Zhejiang, China \\ xiehx887@163.com,31101386@email.zucc.edu.cn
}

\begin{abstract}
Aiming at the problems existed in university computer courses, application-oriented curriculum reform aimed at cultivating the ability of computational thinking was investigated. The quality of teaching will be improved by the teaching reform, if teaching objectives, teaching process and teaching methods were improved. It is the purpose of teaching to improve the computational thinking ability, and to teach the basic ideas and ways of computing disciplines; Teaching process including information processing, typical case studying, and practical training; Using scene and teaching-and-learning environment designed carefully which includes case and task allocation to stimulate students' interest in learning. Furthermore, through combining classroom, laboratory and extracurricular self-taught to form multi-scene, multi-task interaction to achieve teaching objectives.
\end{abstract}

Key words - computational thinking, university computer, teaching case

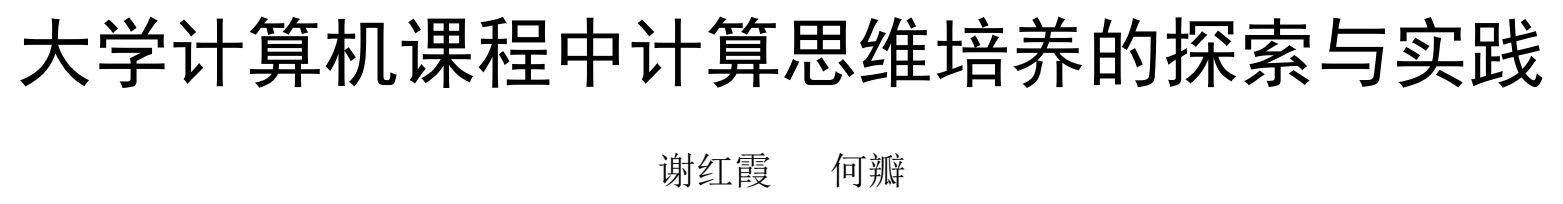

浙江大学城市学院计算机与计算科学学院, 杭州, 浙江, 中国

摘 要 本文针对目前 “大学计算机”课程中存在的问题, 探索面向应用的计算思维能力培养的课程改革, 从教学目标、教学过程、 教学方法几个方面整体推进, 使教学改革对提高教学质量发挥积极作用。明确教学目标为培养计算思维能力, 传授计算学科的基本思 想和思维方式; 教学过程以信息处理过程为主线, 典型案例为导引, 强化实践训练; 教学方法是运用场景式的教学导引方式激发学生 学习兴趣, 通过精心设计教学环境、案例和任务分配, 结合课堂、实验室与课外自学的多场景、多任务联动实现教学目标。

关键词 计算思维, 大学计算机, 教学案例

\section{1. 存在问题}

多年来, 高校中 “大学计算机” 课程作为大学通识课 程, 定位于掌握计算机基础知识, 提高操作技能。由于这 个教学目标设计得太过庞大, 导致教学内容零散, 又限于 课时原因, 每个教学模块都只能机械地搬一大堆的概念进 行解释, 仅仅着眼于告诉学生 “这是什么”, 而不是介绍 “为 什么是这样”, 使课程沦为填鸭式的死记硬背, 把原本应是 丰富有趣, 充满思想启发的课程变得单调乏味, 最终不仅 学生学着无趣, 上课的老师也提不起一点劲, 教学双方都 没有成就感。

其次, 现行的教学模式也存在明显弊端。教学方法沿 用传统的老师讲, 学生听的方式, 信息单向传递, 缺少师
生互动; 课程的考核方式单一, 以期末考试论成绩等等。 结果是学生到课率普遍不高。

在这样的教学状态下, 一些学校不断地压缩课时甚至 取消这门课程也就不足为奇了, 但大学计算机教育的重要 性又不容置疑, 因此如何解决目前的困惑, 改革的出路在 哪里成了大家共同关注的焦点。

\section{2. 研究背景}

2010 年 8 月, 《九校联盟（C9）计算机基础教学发展 战略联合声明》明确提出要旗帜鲜明地把 “计算思维能力 的培养” 作为计算机基础教学的核心任务 [1]。此后, 关于 “计算思维” 的探讨在全国高校持续广泛地进行。2013 年 
8 月, 在第二届 “计算思维与大学计算机课程教学改革研 讨会” 上国家教指委发布了计算思维教学改革白皮书, 白 皮书对高等学校大学计算机课程改革提出了总体思路和指 导意见。

白皮书综合各方面的研究成果, 使用分类的 “核心概 念”方法来构建计算思维的表述体系, 该体系由三层框架, 八类原理组成。首先是 “计算”, 这是第一层次的概念, 其 他概念以 “计算” 为中心并服务于 “计算”。“抽象、自动 化和设计” 为第二层次的概念, 是从不同方面对 “计算” 进行的描述; “通信、协作、记忆、评估” 蕴含在 “抽象、 自动化和设计” 三个概念之中, 是计算机科学中仅次于 “抽 象、自动化和设计” 的基础概念, 对这些概念的理解, 有 助于加深人们对 “计算” 的认知, 具体的表述体系如图 1 所示。白皮书同时给出了大学计算机课程知识体系与核心 概念的关系[2]。

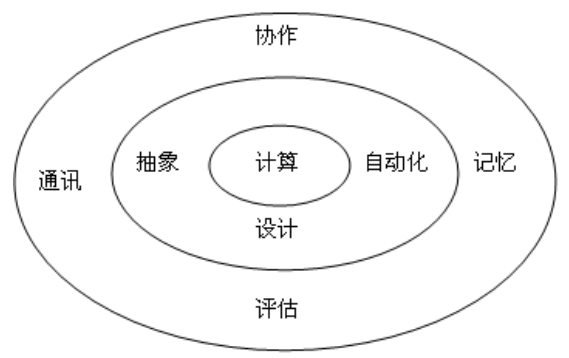

图 1 计算思维表述体系

但是, 白皮书仅是教学改革的总体思路和指导性意见, 要落实其中的精神, 尤其在应用型高校中形成具体的教学 设计方案, 还需要做大量细致的工作。

\section{3. 解决思路}

针对目前的教学现状并根据白皮书的指导精神, 我们 的教学思路是: 面向应用培养计算思维能力。实现路径为: 以信息处理过程为主线, 以典型案例为导引, 强化实践训 练。侧重于计算机学科中的多媒体、网络、数据库等方面 的重点应用来构建教学内容。教学过程中突出计算思维的 基础思想和方法, 通过场景式的教学导引激发学生学习兴 趣、引发积极思考。

在教学实践上, 把体现计算思维特征和方法的知识内 容分解到每一个具体的教学过程中, 通过一堂一堂课程知 识的讲解, 一次一次实践操作的训练, 有目的、有选择地 渗透到教学中, 从而循序渐进地提高计算思维能力。

面向应用的计算思维能力培养并不是对原有教学体系 的全盘否定, 而是继承与创新的结合。计算思维的培养并 不是要代替对于知识和能力的培养, 相反, 它与知识和能 力培养呈现递进的关系。思维的培养必须在知识和能力培
养的基础之上, 而知识和能力的培养必须置身于思维培养 的视野之下，通过讲授计算机的具体理论和技术来揭示有 关计算思维的思想 $[3]$ 。

\section{4. 探索实践}

\section{1 教学研究内容}

教学研究以白皮书中各知识模块和核心概念的关系为 指导, 重点研究如何把核心概念渗透和强化到各知识模块 中, 使得计算思维的核心思想能进一步融入到课程教学中。 围绕这一指导思想重新梳理课程知识体系, 整理教学案例, 设计教学方法, 探索考核手段。

(1) 课程知识体系研究

重新梳理课程教学内容, 围绕计算思维培养, 甄别什 么是需要的, 什么是不需要的, 重视课程间的逻辑联系, 所选择的教学内容要体现计算的原理和核心方法, 同时突 出课程内容的基础性和实践性 ${ }^{[4]}$ 。具体地说, 课程教学内 容以信息处理过程为主线, 包括信息的表示、存储与组织、 计算处理和数据交换等各环节, 并对多媒体、数据库和网 络技术等重点应用进行讲授, 突出计算思维基本方法与实 践应用相结合的特点。教学内容分计算机与计算思维、信 息表示与编码、多媒体技术基础、数据组织与管理、数据 库应用、数据处理算法、计算机网络基础和综合应用八大 教学模块, 涉及信息表示、数字化音视频、计算的过程、 网络搜索引擎及其信息检索、数据安全、数据库应用、简 易编程工具 Scratch 的使用等不同领域的计算机应用。

(2) 教学案例设计

根据理论教学、实践教学和课外自主学习的特点, 研 究不同场景下的典型计算思维教学案例。

理论教学案例结合课内教学内容, 通过案例的引导使 学生了解问题的解决思路, 掌握问题解决的方法和步骤。 如通过 “猜猜我的生日” 引入二进制编码 ${ }^{[5]}$, 通过 “汉诺 塔游戏”引入递归思想方法。

实践教学案例分基本技能训练和应用能力训练。基本 技能训练包括搜索引擎和数字化资源使用、多媒体基础、 办公软件 (Office) 的高级应用等基础性的实践项目。应用 能力训练包括算法设计基础 (Scratch 环境), 数据库 (Access) 应用, 网络设置等面向应用的实践项目。

课外自主学习以预设话题、文献阅读、算法设计等为 主要任务, 在教师引导分析的基础上, 让学生展开课外自 主学习和文献分析等活动, 再将活动成果反馈到课堂教学 或者考核中, 形成课内外教学活动的联动。

(3) 教学方法设计

要将教学内容转化为学生的能力和素质, 教学方法起 着重要的作用。设计以场景导引式的教学方法, 其主要的 
思想是教师如导演, 教案如脚本, 教学环境是舞台, 学生 就是演员。精心设计教学环境、案例和任务分配, 通过课 堂、实验室与课外自学相结合的多场景、多任务配合实现 教学目标。坚持以学生为主体、师生互动和 “做中学”, 既 提升学生的实践应用能力, 也强调解决问题的思路, 训练 计算思维方法。

\section{(4) 考核方法设计}

设计分步式、多样化的面向过程考核方法。考核重心 从 “结果” 考核逐步向 “过程” 倾斜, 使学生认真参与学 习中的每个过程, 如: 课堂讨论、实践环节、课外自主学 习环节等。通过考核时机分步化、考核内容多样化的改革, 使学生注重学习过程和多方面能力的培养。

\section{2 理论教学案例}

如何实现以场景为导引, 通过典型案例来掌握知识, 训练思维? 下面举例来说明。

例: 通过 “汉诺塔游戏” 引入递归算法思想。

教师准备汉诺塔游戏道具, 如图 2 所示 (可以用电子 游戏替代), 从简单的 2 个盘开始玩, 逐个增加盘的数量, 每当盘的数量增加 1 个, 学生觉得移动次数就会增加很多, 当盘的数量为 4 个时, 要移动 15 次才能完成任务, 学生已 经感觉玩得不轻松了。

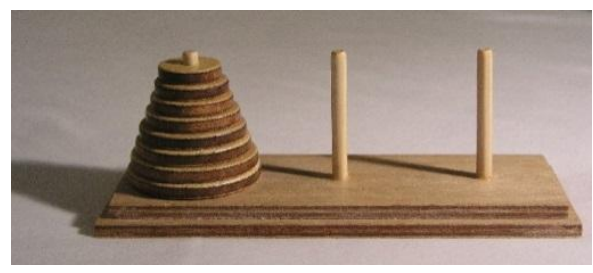

图 2 汉诺塔游戏

然后, 老师通过讲故事的方式介绍这款游戏的来源, 相传在印度有这么一个古老的传说: 在世界中心贝拿勒斯 (在印度北部) 的圣庙里, 一块黄铜板上插着三根宝石针。 印度教的主神梵天在创造世界的时候, 在其中一根针上从 下往上穿好了由大到小的 64 片金片，这就是所谓的汉诺塔。 不论白天黑夜, 总有一个僧侣在按照下面的法则移动这些 金片: 一次只移动一片, 不管在哪根针上, 小片必须在大 片上面。僧侣们预言, 当所有的金片都从梵天穿好的那根 针上移到另外一根针上时, 世界就将在一声霹䨌中消灭, 而梵塔、庙宇和众生也都将同归于尽。学生很好奇这 64 个 盘怎么移呢? 总共要移多少次呢? 这时老师引入递归的思 想, 并把这个思想实现的结果动态演示, 相信学生一定兴 趣盎然，对这个递归的思想方法也会记忆深刻。

设计典型案例, 采用互动的教学方法, 激发学生兴趣, 这是教学取得成功的关键。

\section{3 实践教学案例}

实践教学突出应用性, 关注与理论知识的衔接及具体 实现方法, 由于 Scratch 能让用户创建带交互性的故事、动 画、游戏, 容易入门且实现效果直观有趣, 因此用 Scratch 软件模拟简单算法的实现, 加深学生对计算思想、信息处 理的理解应是最合适的。

例如, 创建 “大鱼吃小鱼” 游戏。一条小鱼在舞台的 随机位置出现, 在 30 秒的时间内, 用鼠标控制大鱼去吃小 鱼。当大鱼吃到小鱼的时候, 发出声音并得分, 并且在舞 台上显示分数及剩余时间。让学生动手玩玩这个小游戏, 看看 30 秒内能拿几分?

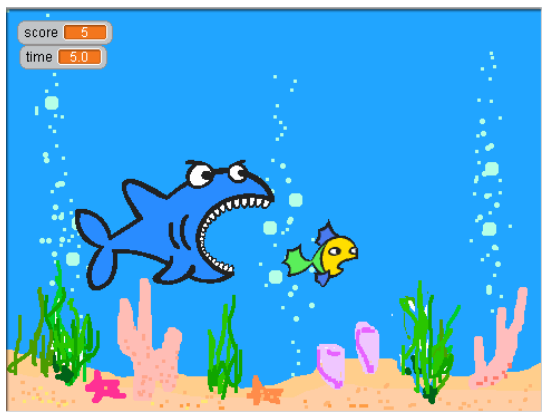

图 3 大鱼吃小鱼演示效果

算法实现的脚本如图 4、图 5 所示, 用一个个现成的 模块搭建, 输入必要的参数即可。只要学生理解了思路, 画出这样的流程图是比较容易的。

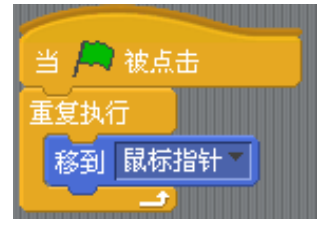

图 4 大鱼实现脚本

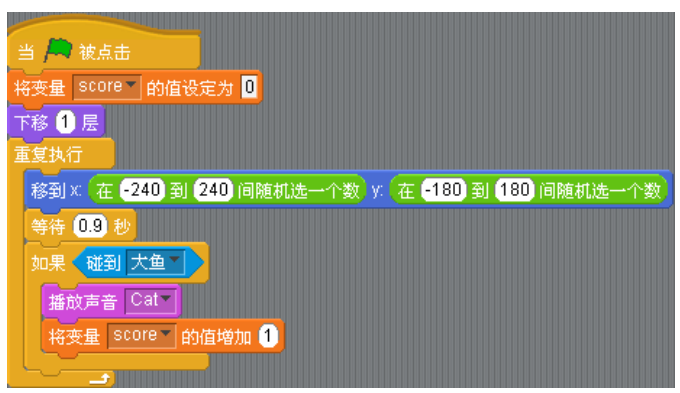

图 5 小鱼实现脚本

用 Scratch 做一些趣味性强的练习, 如 “根据商场促 销方案计算商品实付金额”、“歌手大赛评分”、“求水仙 花数”、“用冒泡算法排序” 等程序。通过一系列的实践 操作便于学生理解计算思维在其中的体现。 
4.4 教学内容设计

以信息处理过程为主线, 以典型案例为导引的 “大学
计算机” 课程, 需要积极探索课程各知识点与计算思维核 心概念的关系, 并把各核心概念渗透和强化到各知识模块 中。表 1 所示为重新梳理和组织后的各章节的教学内容。

表 1 教学内容一览表

\begin{tabular}{|c|c|c|c|}
\hline 章节名称 & \multicolumn{2}{|l|}{ 案例引用 } & 主要内容 \\
\hline $\begin{array}{l}\text { 第 } 1 \text { 章 } \\
\text { 绪论 }\end{array}$ & \multicolumn{2}{|l|}{ 1.网上书店 } & $\begin{array}{l}\text { 计算机的历史, 计算机对社会的影响, 计算机系统组成, 计算与可计算, 计 } \\
\text { 算原理 }\end{array}$ \\
\hline $\begin{array}{l}\text { 第 } 2 \text { 章 } \\
\text { 数制与编码 }\end{array}$ & \multicolumn{2}{|c|}{$\begin{array}{l}1 . \text { 传送紧急情报 } \\
\text { 2.猜姓游戏 } \\
\text { 3.超市购物 }\end{array}$} & $\begin{array}{l}\text { 数制, 数制间转换, 整数的表示, 浮点数的表示, 字符编码, 汉字编码, 条 } \\
\text { 码与编码, 二维码和 RFID, 声音信息, 位图和矢量图, 视频信息, 数据压缩, } \\
\text { 多媒体的编辑 }\end{array}$ \\
\hline $\begin{array}{l}\text { 第 } 3 \text { 章 } \\
\text { 数据的组织 } \\
\text { 与管理 }\end{array}$ & $\begin{array}{l}\text { 1.图书摆放 } \\
\text { 2.营业厅排队 } \\
\text { 3.组织结构图 }\end{array}$ & $\begin{array}{l}\text { 4.地铁线路图 } \\
\text { 5.学生信息管理 } \\
\text { 6.网上书店的数据库设计 }\end{array}$ & $\begin{array}{l}\text { 数据结构, 数据的逻辑结构, 数据的存储结构, 线性表, 栈与队列, 递归, } \\
\text { 树形结构, 图结构, 文件, 文件的结构与存储, 文件目录结构, 文件缓冲区, } \\
\text { 文件操作, 数据管理技术, 数据库, 数据库的三级模式, 数据库的设计, } \\
\text { ACCESS 应用, SQL 语句 }\end{array}$ \\
\hline $\begin{array}{l}\text { 第 } 4 \text { 章 } \\
\text { 数据的处理 } \\
\text { - - 算法 }\end{array}$ & $\begin{array}{l}\text { 1.渡河游戏 } \\
\text { 2.排序算法 } \\
\text { 3.搜索算法 } \\
\text { 4.枚举算法 }\end{array}$ & $\begin{array}{l}\text { 5.递归算法 } \\
\text { 6. 回溯算法 } \\
\text { 7.分治算法 } \\
\text { 8.并行算法 }\end{array}$ & $\begin{array}{l}\text { 算法的概念, 算法的控制结构 (顺序结构、选择结构、循环结构), 算法的表 } \\
\text { 示, 选择排序, 冒泡排序, 直接插入排序, 希尔排序, 快速排序, 归并排序; } \\
\text { 顺序查找, 二分查找, 二叉排序树查找; 枚举算法, 递归算法, 回溯算法, } \\
\text { 分治算法, 并行算法; 可计算性, 计算机处理的局限性, 计算的复杂性 }\end{array}$ \\
\hline $\begin{array}{l}\text { 第 } 5 \text { 章 } \\
\text { 资源共享和 } \\
\text { 信息交换 }\end{array}$ & $\begin{array}{l}\text { 1.匿名的世界 } \\
2 . \mathrm{WiFi} \text { 上网 }\end{array}$ & $\begin{array}{l}\text { 3. 黑屋子里的讨论会 } \\
\text { 4.社会工程学 }\end{array}$ & $\begin{array}{l}\text { 网络的影响, 网络性能, 层次与协议, 网络体系结构, TCP/IP 模型, 数据传 } \\
\text { 送, Internet、Email、FTP、WWW 等服务, 搜索引擎, 微博与微信, Internet } \\
\text { 接入方式, 无线网接入, 加密技术, 数字签名, 防火墙, 社会工程学, 网络 } \\
\text { 安全 }\end{array}$ \\
\hline $\begin{array}{l}\text { 第 } 6 \text { 章 } \\
\text { 简易编程工 } \\
\text { 具: Scratch }\end{array}$ & $\begin{array}{l}\text { 1.小猫打招呼 } \\
\text { 2.沙漠漫步 } \\
\text { 3.猜数大作战 }\end{array}$ & $\begin{array}{l}\text { 4.大鱼吃小鱼 } \\
\text { 5.健康秤 } \\
\text { 6.后缀表达式求值 }\end{array}$ & $\begin{array}{l}\text { Scratch 安装, Scratch 功能, 堆模块, 启动模块, 侦测模块, 动作、外观、声 } \\
\text { 音模块组, 画笔模块组, 变量、数字和逻辑运算模块组, 控制、侦测模块组, } \\
\text { 数学运算, 趣味程序 “健康秤”, 冒泡法排序, 后缀表达式求值, 作品共享 }\end{array}$ \\
\hline
\end{tabular}

\section{5. 后续改革}

“大学计算机” 课程的教学探索和实践任重道远, 有 些方面亟待有所突破。例如如何把计算机基础知识贯彻为 计算思维的核心概念? 如何引导学生突破传统学习方式, 理解这些核心概念, 并建立一定的利用计算机求解问题的 能力。如何在有限的课时之内, 达到上述目标? 这些问题 的解决需要理顺课程内容与计算思维 “核心概念” 的关系, 不断开发优秀的教学案例; 需要教学方法和手段的配合; 需要进一步完善信息化教学平台, 引入微视频、动画, 加 强教学过程管理等; 需要引导和发展课外学习、课外实验、 课外阅读、作业等; 需要利用考核这个指挥棒, 进一步改 进考核方法, 减少笔试的分量, 加强实验报告、课程讨论 等的比重。

教学改革的步子已经迈出, 只有不断探索, 勇于实践, “大学计算机” 教学才能迎来新的生机。

\section{参考文献(References)}

[1] Nine college alliance (C9) Computer-based teaching strategy Joint Statement. China University Teaching.2010(9)

[2] Computational Thinking Teaching White Paper. The Ministry of education of University Computer Teaching Guidance Committee.2013

[3] Declaration of Computational Thinking Teaching Reform. The Ministry of education of University Computer Teaching Guidance Committee.2013

[4] He QinMing, Lu HanQuan, Feng BoQin. The Core Task Of Basic Computer Teaching Is Cultivating Computational Thinking Ability. China University Teaching.2010(9)

[5] Tim Bell Et al. Sun Junfeng, Yang Fan Translation. Computer Science Unplugged. Wuhan: Huazhong University of Science and Technology Press, 2010. 